\title{
Optical and electric properties of dynamic holographic gratings with arbitrary contrast
}

\author{
Kukhtarev, Nickolai; Buchhave, Preben; Lyuksyutov, Sergei
}

Published in:

Physical Review A

Link to article, DOI:

10.1103/PhysRevA.55.3133

Publication date:

1997

Document Version

Publisher's PDF, also known as Version of record

Link back to DTU Orbit

Citation (APA):

Kukhtarev, N., Buchhave, P., \& Lyuksyutov, S. (1997). Optical and electric properties of dynamic holographic gratings with arbitrary contrast. Physical Review A, 55(4), 3133-3136. https://doi.org/10.1103/PhysRevA.55.3133

\section{General rights}

Copyright and moral rights for the publications made accessible in the public portal are retained by the authors and/or other copyright owners and it is a condition of accessing publications that users recognise and abide by the legal requirements associated with these rights.

- Users may download and print one copy of any publication from the public portal for the purpose of private study or research.

- You may not further distribute the material or use it for any profit-making activity or commercial gain

- You may freely distribute the URL identifying the publication in the public portal 


\title{
Optical and electric properties of dynamic holographic gratings with arbitrary contrast
}

\author{
N. V. Kukhtarev, ${ }^{*}$ P. Buchhave, and S. F. Lyuksyutov ${ }^{\dagger}$ \\ Department of Physics, Technical University of Denmark, DK2800 Lyngby, Denmark
}

(Received 29 May 1996)

\begin{abstract}
An analytical solution of the photoconductive material equations for dynamic holographic gratings of arbitrary contrast has been obtained. A method of measuring high-contrast correlation functions has been suggested and tested experimentally. Good agreement with the analytical expression for the current at high contrast and experiment is found. An interesting effect of the holographic transversal photocurrent is predicted and a number of high-contrast-related topics are discussed. [S1050-2947(97)01704-6]
\end{abstract}

PACS number(s): 42.40.Eq, 42.65.-k

Dynamic photoexcited gratings (PG's) are widely used for characterization of materials, for signal processing and for information storage [1]. Recently, a surge of interest in PG's was inspired by the discovery of highly efficient materials for grating recording, e.g., photorefractive polymers [2] and photorefractive liquid crystals [3], and by the observation of photoconductivity in rare-earth-doped laser crystals [4].

Effects due to the nonlinearity of the material equations are strongly enhanced with increasing contrast of the light interference pattern. Early solutions of the material equations of the photogeneration-diffusion-drift-trapping (PDDT) model, also called the band transport model [5], were valid only for gratings of small contrast (linearized solutions). Extensions of the linear solution have been obtained including quadratic [6] and cubic [7] expansion in terms of the contrast $m$, and numerical solutions have also been obtained [8]. However, these approaches suffered from analytical and computational complexity. The latest attempt [9] at the solution of the PDDT model gives results valid only for the diffusion-dominated case, and does not include a description of photoconductive gratings. A "high-contrast correction" was introduced in the description of ferroelectrics $[10,11]$ and also for semiconductors $[10,12,13]$, and experimental results of the harmonic content of the space-charge field has been reported in [14]. The electric current along the grating vector is proportional to $\left(1-m^{2}\right)^{1 / 2}$ in the small-contrast approximation. In the case of high contrast, when $m \rightarrow 1$, the current according to this expression must vanish in contradiction to experimental results. To account for the experimental results empirical "artificial" correction functions for the amplitude of holographic grating as a function of the modulation of the light interference pattern have been suggested $[6,15]$ in an attempt to describe the high-contrast case. In this paper we present a simple analytical solution for the PDDT model, giving the spatial distribution of photoconductivity, space-charge field, and ionized donors. The solution is based on a simple but profound observation from numerical simulations of the full set of PDDT equations [17] regarding

\footnotetext{
*Permanent address: Physics Department, Alabama A\&M University, Normal, AL 35762.

†Permanent address: Institute of Physics, National Academy of Sciences, 252650 Kiev, Ukraine.
}

the form of these variables as a function of the spatial coordinate in direction of the grating vector: Whereas the spacecharge field and the ionized donor density have a highly nonlinear relation to the "driving force," the light intensity pattern impressed on the crystal, the free-carrier distribution contains a negligible amount of higher harmonics. This fact allows us to find a simple analytical solution for the freecarrier distribution for an arbitrarily high index of modulation of the impressed light distribution. This in turn leads to a simple analytical expression for the high-contrast correction (also called the reduction factor). Since the other variables may be expressed directly in terms of the carrier distribution, we can now find simple Fourier expansions for the space-charge field and the ion density. The ability to express the spatial variation of the physical quantities in simple analytical equations for arbitrary contrast has great significance for the understanding of the photorefractive process and for the prediction of experiments and applications, and, since the PDDT model has applications or equivalents in other photoinduced processes, we expect that some of these results may have importance in other fields as well.

We consider illumination with a sinusoidal light intensity grating:

$$
I=I_{0}(1+m \cos (K x)),
$$

where the contrast $m$ can have any value between 0 and $1 . I_{0}$ is the total intensity of incident light, $K$ is the grating vector, and $x$ is the spatial coordinate along the grating normal. The PDDT model $[5,16]$ provides the following relations for the total current $J$, the space-charge field $E_{\mathrm{sc}}$, and the photocarrier concentration $n$ assuming linear recombination and negligible depletion of photoactive impurity centers:

$$
\begin{gathered}
J=e \mu\left\langle n^{-1}\right\rangle^{-1} E_{0}, \\
E=E_{D}(\ln n)^{\prime}+E_{0}\left(n\left\langle n^{-1}\right\rangle\right)^{-1}, \\
\lambda(\ln n)^{\prime \prime}+\xi\left(n^{-1}\right)^{\prime}\left\langle n^{-1}\right\rangle^{-1}=1-\frac{N^{+}}{N_{A}}
\end{gathered}
$$

where $N^{+}=\left(N_{A} / n\right) G$ is the density of charged ions, $E_{D}=(D / \mu) K$ is the diffusion field, $E_{0}$ is the external applied electric field along the grating vector, $\mu$ is the mobility of carriers, $D$ is the diffusion constant, $N_{A}$ is the concentration of acceptors, $\lambda=E_{D} / E_{Q}$, and $\xi=E_{0} / E_{Q}$. The saturation field 
$E_{Q}$ equals $N_{A} /\left(\varepsilon_{0} \varepsilon K\right)$, where $\varepsilon_{0}$ is the free-space permittivity, and $\varepsilon$ is the static dielectric constant. The derivative is taken with respect to $u=K x$. The generation function $G$ is related to the intensity pattern (1) as $G=G_{0}(1+\bar{m} \cos u)$, where $G_{0}=\left(\beta_{d}+\beta_{i}+s I_{0}\right)\left(N-N_{A}\right) /\left(\gamma N_{A}\right), \beta_{d}$ is the rate of photocarriers generation in the dark, $\beta_{i}$ is the generation rate under incoherent light related to scattering and reflection inside the material, $\bar{m}=m /\left(1+\left(\beta_{d}+\beta_{i}\right) / s I_{0}\right)$ is the contrast reduced due to dark conductivity and the influence of incoherent scattering, and \langle\rangle indicates spatial averaging.

Looking for periodic solution of Eq. (4) in the form

$$
n=n_{0} \sum_{p}\left(a_{p} \cos p u+b_{p} \sin p u\right),
$$

with $a_{0}=1$, and assuming that high harmonics are negligible, we obtain the fundamental harmonic solution after averaging Eq. (4) and calculating coefficients for $\cos u$ and $\sin u$. Averaging Eq. (4) means that we select a solution that satisfies conditions of neutrality both integrally over one period and locally for the fundamental harmonic. As a result we find $n_{0}$, $a_{1}$, and $b_{1}$, or equivalently $n_{0}, a$, and $\varphi$, where $a_{1}=a \cos \varphi$, $b_{1}=-a \sin \varphi$, and $\tan \varphi=-\xi /(1+\lambda)$ :

$$
\begin{gathered}
a=\frac{M}{1+(M \delta)^{2}}\left[1-\delta \sqrt{1+M^{2}\left(\delta^{2}-1\right)}\right], \\
\frac{n_{0}}{G_{0}}=\frac{M}{a(1+\lambda)} .
\end{gathered}
$$

Here $\delta=\lambda /(1+\lambda)$ and $M=\bar{m}(1+\lambda) / \sqrt{(1+\lambda)^{2}+\xi^{2}}$. The physical meaning of $\varphi$ is a phase shift between the light pattern and the carrier grating, which is clear if we rewrite the expression for the fundamental harmonics of $n$ in the following form:

$$
n_{1}=n_{0}\left(1+a_{1} \cos u+b_{1} \sin u\right)=n_{0}(1+a \cos (u+\varphi)) .
$$

For the evaluation of the higher harmonics, we rearrange Eq. (5) in the following form:

$$
n=n_{0}(1+a \cos (u+\varphi)+f),
$$

and for $f$, which is simply the sum of higher harmonics, we use an iteration approach based on placing linear terms in Eq. (4) on the left-hand side and nonlinear terms on the right-hand side. Neglecting $f$ in all nonlinear terms, we obtain the following equation:

$$
\lambda\left(\frac{n_{1}}{n_{0}}+f\right)^{\prime \prime}-\left(\frac{n_{1}}{n_{0}}+f\right)=\frac{\lambda\left(n_{1}^{\prime}\right)^{2}}{n_{0} n_{1}}+\frac{n_{1}^{\prime} \xi \sqrt{1-a^{2}}}{n_{1}}-\frac{G}{n_{0}} .
$$

The procedure for calculating the higher harmonics analytically is relatively simple. Below we give the expression for the second harmonic:

$$
n_{2}=n_{0}\left(\overline{a_{2}} \cos [2(u+\varphi)]+\overline{b_{2}} \sin [2(u+\varphi)]\right)
$$

where

$$
\overline{a_{2}}=\frac{2 \lambda\left(1-\sqrt{1-a^{2}}\right) \sqrt{1-a^{2}}}{(4 \lambda+1) a^{2}},
$$

and $\overline{b_{2}}=-(\xi / \lambda) \overline{a_{2}}$. Analysis of the condition $a \gg \overline{b_{2}}, \overline{b_{2}}$ shows that it is valid for the whole range of parameters. This point justifies the validity of the solution for the fundamental harmonic in form (6) for small as well as high contrast $m$.

Equation (9) with $n_{0}, a$, and $\varphi$ given by Eqs. (6) and (7) provides the desired analytical solution for the carrier concentration for arbitrary value of the light intensity modulation, $m$, without using the small modulation assumption or an assumed functional form for the carrier distribution. We can obtain a simple expression for the case of small contrast, $M^{2}\left(\delta^{2}-1\right) \ll 1$, by using the general solution (6). We find

$$
a \simeq m R\left[1+\frac{\lambda}{2}(m R)^{2}\right]
$$

where

$$
R=\left\{\left(1+\frac{\beta_{d}+\beta_{i}}{s I_{0}}\right) \sqrt{(1+\lambda)^{2}+\xi^{2}}\right\}^{-1}
$$

is the reduction factor first introduced in [16], and recently investigated by using cross talk between two slightly different gratings in photorefractive BSO [18]. Note that the first nonlinear term in the expression for the contrast $m$ is cubic, which reflects the fact that $a(m)$ is a nonsymmetric function in solution (6). For high contrast when $\delta^{2} \gg\left(1-M^{2}\right)\left(1-\delta^{2}\right)$, from Eq. (6) we obtain

$$
a=\frac{M\left(1-\delta^{2}\right)\left(1+M^{2}\right)}{2\left(1+(M \delta)^{2}\right)} .
$$

Having obtained the solution for $n$, the spatial distribution of the space-charge field $E(x)$ and the concentration of ionized donors $N^{+}$can also be found from the basic equations (2)-(4). The electric current is an explicit function of the contrast,

$$
J=e \mu n_{0} E_{0} \sqrt{1-a^{2}},
$$

where $a$ can be close to 1 , and significantly change the current at high values of the contrast $m$. Thus current measurements provide an interesting tool for verifying the present theory and for sensing the condition of the carrier grating, especially at high contrast. As an example, knowledge of the analytical dependence of $a$ on the contrast $m$ gives the degree of coherence or the correlation function of the interacting beams. Thus we may use relation (14) in the case of a BSO crystal for determination of the important Debye screening length $[1,4]$, and also for the study of the influence of scattering and other sources of incoherence, which may reduce the modulation of the carriers.

For the electric field and its spatial Fourier harmonics, from Eqs. (3) and (5) we obtain 


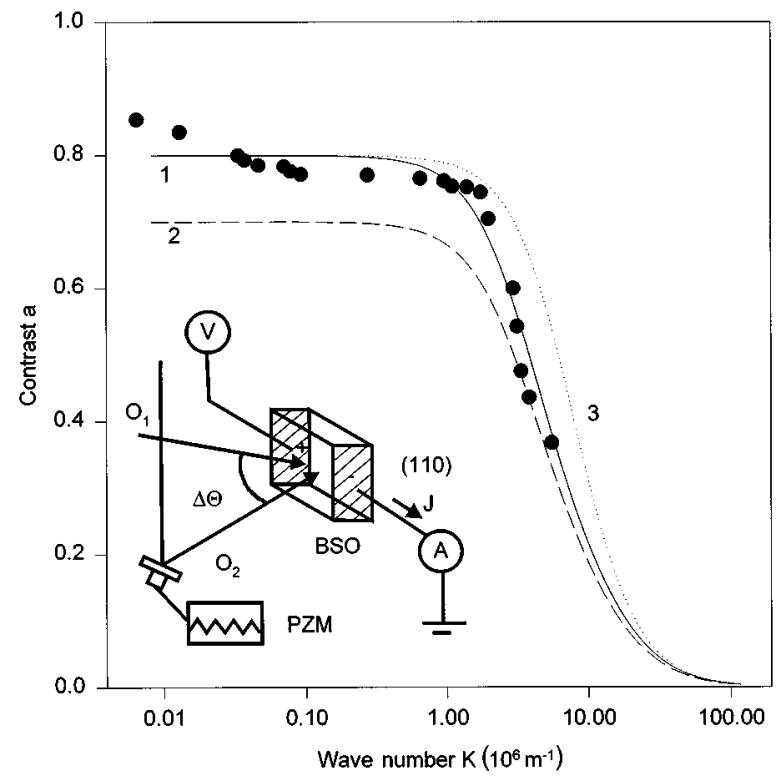

FIG. 1. Black circles correspond to experimental points for applied voltage $5 \mathrm{kV}$ and $m=1$. Solid curves calculated from Eq. (6), curve 1 is for $\Lambda_{d}=0.77 \mu \mathrm{m}, \bar{m}=0.8$; curve 2 for $\Lambda_{d}=0.77 \mu \mathrm{m}$, $\bar{m}=0.7$; and curve 3 for $\Lambda_{d}=0.3 \mu \mathrm{m}, \bar{m}=0.8$.

$$
\begin{aligned}
E & =\frac{E_{0} \sqrt{1-a^{2}}-E_{D} a \sin (u+\varphi)}{1+a \cos (u+\varphi)} \\
& =\frac{1}{2} \sum_{p=1,2, \ldots}\left(\left(E_{0}-i E_{D}\right) A^{(p)} e^{i(u+\varphi) p}+\text { c.c. }\right),
\end{aligned}
$$

where

$$
A=\frac{\sqrt{1-a^{2}}-1}{a}=\frac{\sqrt{1+M^{2}\left(\delta^{2}-1\right)}-1}{M(1+\delta)}
$$

is the amplitude of the fundamental harmonic, which for small modulation $m$ takes the form

$$
A=-\frac{M}{2(1+\lambda)}\left\{1+\frac{M^{2}}{4}\left(1-\delta^{2}\right)\right\} .
$$

For $\mathrm{N}^{+}$we obtain the following expression:

$$
N^{+}=\frac{N_{A} G}{n}=\frac{a(1+\lambda) N_{A}(1+\bar{m} \cos u)}{M[1+a \cos (u+\varphi)]} .
$$

The amplitude of the $E$-field grating [Eqs. (17) and (18)] is the starting point for the calculation of beam coupling and diffraction efficiency in photorefractive materials. From the general expression Eq. (14) we can obtain the results, presented in $[10]$, for the special case of $E_{0}=0(\xi=0)$ and $\lambda \equiv\left(k_{D} / k_{Q}\right)^{2} \ll 1$.

The experimental configuration is sketched in Fig. 1 [18]. Two plane-wave beams $O_{1}$ and $O_{2}$ from a single-mode Arion laser polarized in the plane of incidence and separated by an angle $\Delta \theta$ are incident on a sample of photorefractive BSO. The (110) surface is perpendicular to the wave vector $K$ of the grating. The fringe spacing was chosen in the range $0.5-900 \mu \mathrm{m}$. The external electric voltage $E_{0}=5 \mathrm{kV}$ is applied along the grating wave vector. We measure the current

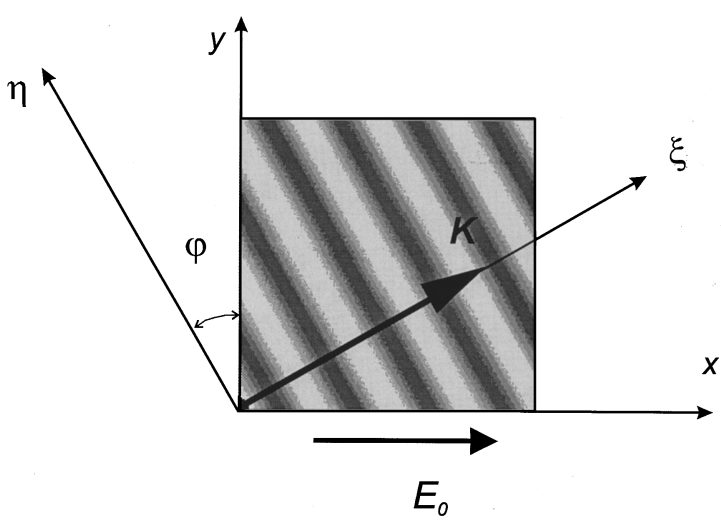

(a)

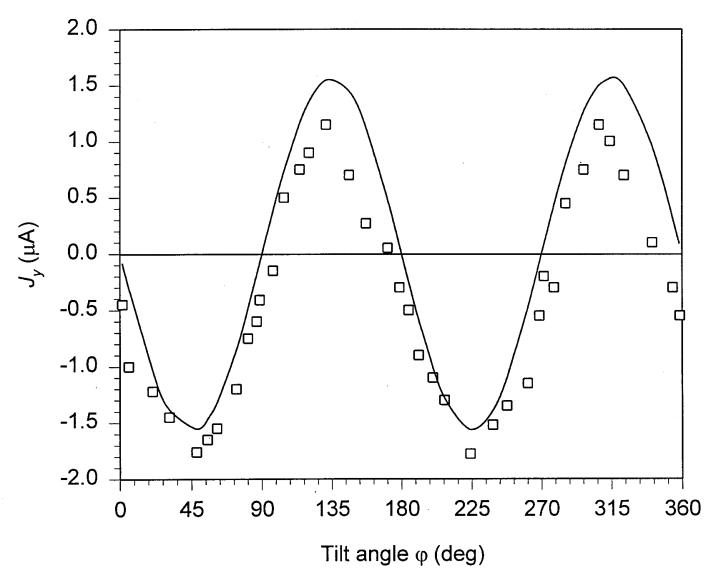

(b)

FIG. 2. (a) Orientation of tilted interference pattern for formation anisotropic current in the $y$ direction. (b) Dependence of the anisotropic current $j_{\perp}$ on angle $\varphi$. Open squares: measurement; solid curve: theory.

between the negative connection to the sample and ground. A phase modulation is applied to beam $\mathrm{O}_{2}$ with a piezoelectric mirror driven by a sine voltage, resulting in a harmonic motion of the optical interference pattern inside the crystal. By this means the photorefractive grating can be switched on and off without changing the total intensity of the incident light. According to formula (15), the current must have its maximum value when there is no modulation of the light incident on the sample, i.e., when the contrast $a$ equals zero. We then have $J=J_{\max }=e \mu n_{0} E_{0}$. Modulation of the incident light results in modulation of the free-carrier distribution, leading to a decrease of the photoconductivity and of the measured current. Thus by measuring the current with and without modulation of the incident light we can find the ratio $J / J_{\max }=\sqrt{1-a^{2}}$, which gives us the value of the modulation of the carrier distribution $a$, and, in general, information about the reduction factor $R$, because $a=m R$. For $m=1$, the value of $a$ is simply equal to $R$, where $R$ itself depends on the modulation of light $m$.

The experimental dependence of the contrast $a$ of the carrier grating on the wave number $k$ of the interference fringes is shown in Fig. 1. The wavelength for the experiment was selected to be $488 \mathrm{~nm}$. The theoretical dependence (solid curve 1) is calculated from Eq. (6) using the following parameters valid for BSO: $\Lambda_{d}=0.77 \mu \mathrm{m}, \bar{m}=0.8$, and $E_{0}=5$ $\mathrm{kV} / \mathrm{cm}$. Attempting to create a good fit to the measured points using the existing theory by choosing other values for 
$\bar{m}(\bar{m}=0.7$, curve 2$)$, and for $\Lambda_{d}\left(\Lambda_{d}=0.30 \mu \mathrm{m}\right.$, curve 3$)$ fail by giving a different functional form. The discrepancy for small values of $k$ between experiment and theory may be explained by the growing contribution from multiple reflections (Fabry-Pérot modes) for small incident angles. This leads to an increase in incoherent scattering, decrease of contrast and increase of holographic current (in accordance with the experiment).

This experiment allows a direct measurement of the contrast $a$ of the carrier distribution in the sample without using the photorefractive effect. This is a matter of importance, because the redistribution of carriers in a photoconductive sample is determined mainly by the recombination time and not on the Maxwell relaxation time, which is important in the photorefractive effect. Thus current measurements as described above may, in the case of photoconductive samples, lead to fast correlators.

The present solution may, due to its simple analytical form, throw light on a great number of interesting problems. As examples we may mention that we find a slowdown of the grating build-up time with increasing contrast. Such a slowdown was reported recently in a numerical study [19]. Further we may mention the study of beam-coupling effects. Using the expressions for $n_{0}, a$, and $\varphi$ we can obtain a solution for the intensities of the coupled waves in both photoconductive and photorefractive samples. In the case of photoconductive samples, we find that the direction of energy transfer depends on the sign of the external electric field $E_{0}$. Sign sensitivity of the energy transfer may be useful in some applications such as visualization of near-contact elec- tric field distribution and of charged defect clusters in semiconductors. These findings will be reported in a separate publication.

This solution described above, which was derived for external field $E_{0}$ along the grating vector $K$, has been generalized for a tilted grating, where $E_{0}$ and $K$ form angle $\varphi$, Fig. 2(a). For a tilted geometry we predict appearance of a transverse current, which can be detected by an additional pair of electrodes. Detailed calculations of this effect will be presented in [20]. Here we give a formula for the dependence of the transverse current $j_{\perp}$ on angle $\varphi: j_{\perp}=\left(E_{0}\left(\sigma_{\perp}\right.\right.$ $\left.\left.-\sigma_{\|}\right) \sin 2 \varphi\right) / 2$, where $\sigma_{\perp}=\sigma_{\|} \sqrt{1-a^{2}} ; \sigma_{\perp}=e \mu n$. This effect can be very useful for processing of both optical and electrical signals. It resembles the Hall effect, but does not require a magnetic field. Preliminary experiments in sillenites show good agreement with the predicted dependence of $j_{\perp}$ on the intersection angle $\varphi$ [Fig. 2(b)].

In conclusion, we solved the material equations of the PDDT model for periodic modulation of photoexcitation with arbitrarily high contrast. This solution allows the experimental determination of Debye screening length and the reduction factor related to incoherence factors in BSO samples by a very simple method based on measurement of steady-state current. This high-contrast solution can also be used for the interpretation of the measured correlation function of interacting beams with high degree of coherence.

S.L. acknowledges the support from Danish TechnicalScientific Research Council Grant No. 9400102. N.V.K. acknowledges the support from U.S. Army SSDC under Contract No. DASG 60-95-2-00.
[1] M. P. Petrov, S. I. Stepanov, and A. N. Khomenko, in Photorefractive Crystals in Coherent Optical Systems (Springer, New York, 1991).

[2] S. Ducharme, J. C. Scott, R. J. Tweig, and W. E. Moerner, Phys. Rev. Lett. 66, 1846 (1991).

[3] I. C. Khoo, Opt. Lett. 20, 2137 (1995).

[4] M. A. Noginov, N. Kukhtarev, N. Noginova, H. J. Caulfield, P. Venkateteswarlu, and M. Mahdi, in OSA Proceedings on Advanced Solid-State Lasers (OSA, Washington, DC, 1996), Vol. 18, p. 323.

[5] N. Kukhtarev, Pis'ma Zh. Tekh. Fiz. 2, 1114 (1976) [Sov. Tech. Phys. Lett. 2, 436 (1976)].

[6] Ph. Refregier, L. Solymar, H. Rajbenbach, and P. Huignard, J. Appl. Phys. 58, 45 (1985).

[7] R. Saxena and T. Y. Chang, J. Opt. Soc. Am. B 9, 1467 (1992); B. Y. Zel'dovich and E. P. Shershakov, Quantum Electron. 24, 68 (1994).

[8] F. Vachss and L. Hesselink, J. Opt. Soc. Am. B 5, 1814 (1988).

[9] E. Serrano, M. Carrascosa, and F. Augullo-Lopez, Opt. Lett. 20, 1910 (1995).

[10] V. Vinetskii and N. Kukhtarev, Fiz. Tverd. Tela (Leningrad)
16, 1895 (1974) [Sov. Phys. Solid State 16, 2414 (1975)].

[11] A. Krumins and P. Gunter, Phys. Status Solidi A 63, K111 (1981).

[12] V. Haken, M. Hunhaussen, and L. Ley, Phys. Rev. B 51, 10579 (1995).

[13] D. Ritter, E. Zeldov, and K. Weser, J. Appl. Phys. 62, 4563 (1987).

[14] Y. P. Shershakov, Yu. V. Miklyaev, and B. Y. Zel'dovich, Appl. Phys. A 59, 575 (1994).

[15] G. H. Kwak, J. Takacs, and L. Solymar, Opt. Commun. 96, 278 (1992).

[16] N. Kukhtarev, V. Markov, S. Odulov, M. Soskin, and V. Vinetskii, Ferroelectrics 22, 949 (1979).

[17] As suggested on the basis of numerical simulations, P. Buchhave (private communication).

[18] P. E. Andersen, P. Buchhave, P. M. Petersen, and M. V. Vasnetsov, J. Opt. Soc. Am. B 12, 1422 (1995).

[19] A. Bledowski, J. Otten, and K. H. Ringhofer, Opt. Lett. 16, 672 (1991).

[20] N. Kukhtarev, S. Lyuksyutov, P. Buchhave, H. J. Caulfield, and M. Vasnetsov, Opt. Lett. (to be published). 